EPJ Web of Conferences 41, 10018 (2013)

DOI: $10.1051 /$ epjconf/20134110018

C) Owned by the authors, published by EDP Sciences, 2013

\title{
Strong Field Acceleration of Attosecond Electron Pulses emitted by a Sharp Metallic Nanoprobe
}

\author{
D. J. Park ${ }^{1,2}$, S. Schmidt ${ }^{1,2}$, B. Piglosiewicz ${ }^{1,2}$, and C. Lienau ${ }^{1,2}$ \\ ${ }^{1}$ Institut für Physik, Carl von Ossietzky Universität, 26129 Oldenburg, Germany \\ ${ }^{2}$ Center of Interface Science, Carl von Ossietzky Universität, 26129 Oldenburg, Germany
}

\begin{abstract}
We report on the observation of strong near-field acceleration of attosecond electron pulses emitted from a sharp nanometer-sized gold tip. Kinetic energy spectra extending over tens of $\mathrm{eV}$ and varying qualitatively with laser wavelength and intensity are explained in terms of the spatiotemporal electron dynamics in the strong field gradient at the tip apex.
\end{abstract}

\section{Introduction}

Recently, the optically induced emission of ultrafast electron bursts from single metallic nanostructures attracts substantial interest $[1,2]$. Due to the high-order nonlinearity of the emission process, such electron bursts are expected to have durations much shorter than a single field cycle. Hence such laser-illuminated tips are believed to present an interesting source for future generation attosecond electron microscopes and their physical properties are intensely studied. Very recently, first studies of the effect of the carrier-envelope phase of few cycle laser pulses on the kinetic energy of ejected electrons have been reported, indicating the possibility to steer electron dynamics by strong field acceleration on attosecond time and nanometer length scales [3]. So far, however, strong-field effects remained rather weak and details of the spatio-temporal dynamics unknown.

Here, we study the effect of the laser wavelength and pulse energy on the energy spectrum and angular distribution of tip-enhanced electron emission. We observe striking, plateau-like energy spectra, extending over tens of $\mathrm{eV}$ and depending sensitively on laser wavelength and intensity. We argue that these spectra provide new and unprecedented insight into dynamics of the electron generation and acceleration process.

\section{Details}

Angle-resolved kinetic energy spectra of electrons emitted from sharp, chemically-etched gold tips irradiated with 25-fs-laser pulses derived from a noncollinear optical parametric amplifier and tunable between $800 \mathrm{~nm}$ and $1.5 \mu \mathrm{m}$ are investigated. To avoid tip damage, the incident pulse energy was kept below $1.3 \mathrm{~nJ}$, corresponding to a peak intensity of $\sim 2 \times 10^{12} \mathrm{~W} / \mathrm{cm}^{2}$. Angle-resolved kinetic energy spectra are recorded with a hemispherical photo-electron-spectrometer (PES) equipped with a two-dimensional CCD detector. 
Figs. 1 (a) and (b) show kinetic energy spectra for two different laser wavelength of $1100 \mathrm{~nm}$ (a) and $1400 \mathrm{~nm}$ (b) as a function of pulse energy. Spectra at $1100 \mathrm{~nm}$ show a simple exponential decay at high energies, originating mainly from multiphoton ionization and above threshold ionization [1,3]. Spectra at $1400 \mathrm{~nm}$, however, show, in the high-intensity limit, a pronounced plateau-like spectrum extending over more than $20 \mathrm{eV}$. The spectral line shape varies qualitatively with intensity, giving the usual exponential decay at low intensities. These high-intensity plateaus resemble those known from high harmonic generation, yet have a much higher relative intensity and are much more pronounced. A clear high-energy cut-off is observed and it is seen that the low-energy wing of the spectrum shifts strongly with intensity. Kelysh parameters of less than 0.5 are deduced.

(a)

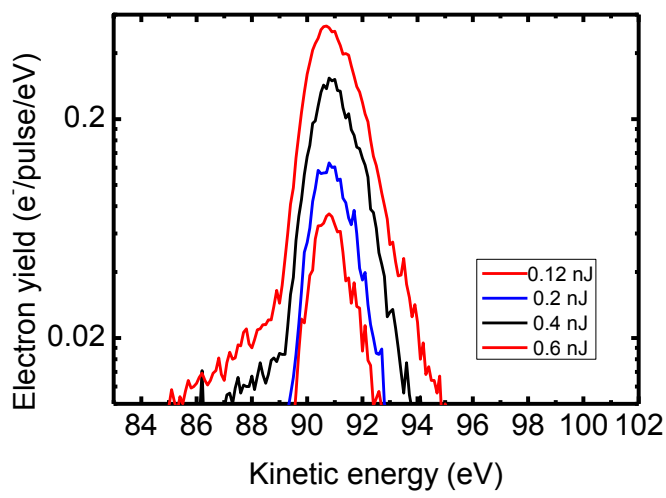

(b)

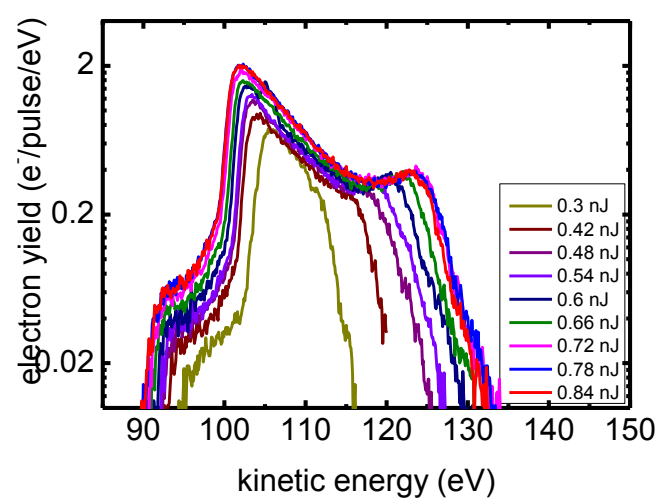

Fig. 1. Kinetic energy spectra recorded as a function of laser pulse energy, varied between $0.12 \mathrm{~nJ}$ and $0.84 \mathrm{~nJ}$ for center wavelengths of $1100 \mathrm{~nm}$ (a) and $1400 \mathrm{~nm}$ (a). The insets show the spectrally integrated electron yield as a function of laser pulse energy, plotted on a double logarithmic scale. A clear plateau in the energy spectrum is observed in the long-wavelength $(1400 \mathrm{~nm})$ and high pulse energy limit.

To understand these pronounced changes in the kinetic energy spectra, we analyze the dynamics of the electron generation and acceleration within the oscillating laser field confined to the near field region of the tip with the help of simulation based on a modified two-dimensional Simpleman's model [3]. In general, the oscillating electric field induces a quiver motion of the electron [4]. In case of a spatially homogeneous field, acceleration during the negative half cycle and deceleration during the positive half cycle effectively cancel out and the net, time-averaged force excerted by the light field on the electron is small. In our experiment, however, large field enhancement at the very apex of the gold tip induces a strong field gradient on a $20 \mathrm{~nm}$ length scale. Hence, an electron being accelerated away from the tip during the negative half cycle will move to a region of reduced field strength and therefore experience a reduced back-acceleration during the subsequent positive half cycle. This results in a strong acceleration of electron. Evidently, the electron trajectories within the tips' near field depend sensitively on the ionization time of the electron with respect to the absolute phase of the laser. This is schematically depicted in Fig 2. (a) showing the trajectories of two electrons born with a relative phase delay of only $\sim 1 / 4$ of a full cycle, i.e., with a time difference of only 800 as in case of $1400 \mathrm{~nm}$ wavelength. Evidently, the resulting net kinetic energy of the electrons varies distinctly. Hence, a simultaneous measurement of electron kinetic and angular distribution provides direct insight into the spatiotemporal trajectories of the electrons during acceleration within the surface-confined, temporally oscillating electric field gradient. In such experiments we find, as expected from the dipolar shape of the tips' near field, that low energy electrons are emitted into a large opening angle of more than $30^{\circ}$. This is fundamentally different for the fast electrons with kinetic energies exeeding $10 \mathrm{eV}$. Here we observe a clear reduction in 
emission angle - the fast electrons are steered within the near field towards the tip axis. The underlying physics is illustrated in Fig. 2(b), depicting a vectorial plot of the instantaneous electric field in the vicinity of the tip together with the trajectories of two electrons born at the same position in space but with a time delay of 800 as. The steering of the fast electron towards the tip axis is clearly visible. It seems quite remarkable that such a pronounced variation in emission angle and kinetic energy is achieved within a local field confined to few tens of nanometers only. This implies that such angle- and energy resolved measurments can be a powerful tool for analyzing the spatiotemporal dynamics of electrons on nanometer length and femtosecond time scales.
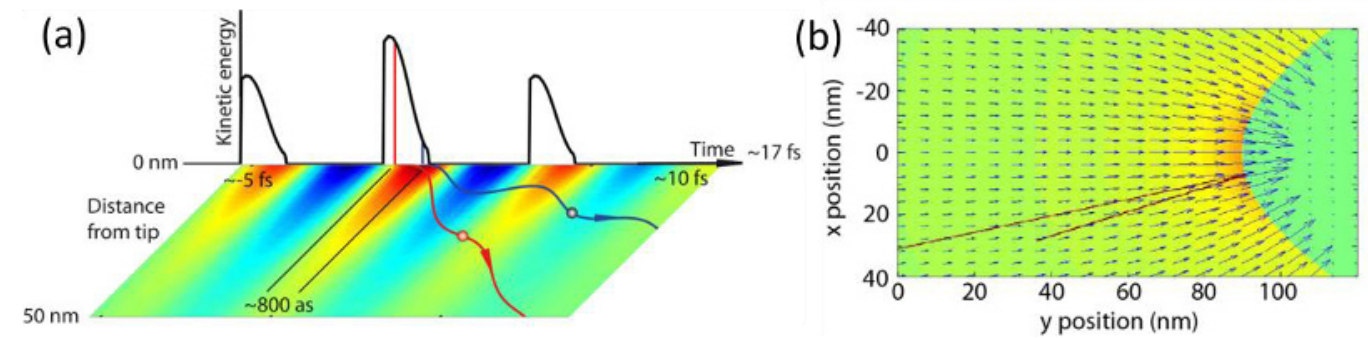

Fig. 2. (a) Schematic diagram of the electron trajectories for two different birth times. The color code gives the spatiotemporal dynamics of the electric field of the laser in the vicinity of the tip together with a simulation of the real space motion of the electron driven by the laser field on an attosecond time and nanometer length scale. The simulated terminal kinetic energy as a function of its birth time is shown as a black solid line. (b) Vectorial map of the near field in the vicinity of a gold tip with $10 \mathrm{~nm}$ radius of curvature together with simulated trajectories of two electrons born at the same position but with a time delay of 800 as. Strong-field steering of the electron dynamics within the near field of the tip is clearly visible.

\section{Conclusions}

In conclusion, we have observed pronounced plateaus in electron kinetic energy spectra emitted from the apex of sharp gold tips under intense ultrashort pulse irradiation. Strong changes in spectral shape with pulse intensity and wavelength reflect different strong field acceleration dynamics of attosecond electron bursts emitted from such tips. Analysis of kinetic energy and angular dispersion of these electrons can give fundamentally new insight into the light-driven electron dynamics on attosecond time and nanometer length scale and might pave the way towards electron microscopy with attosecond time resolution.

\section{References}

1. C. Ropers et al., Physical Review Letters, 2007. 98: p. 43907.

2. R. Bormann et al., Physical Review Letters, 2010. 105: p. 147601.

3. M. Krüger et al., Nature, 2011. 475: pp. 78-81.

4. G. Herink et al., Nature, 2012. 483: pp. 190-193 